\title{
Automatic recognition method of doors and windows fan area based on
}

\section{convex hull algorithm}

\author{
Kai Dong ${ }^{1, a}$, Xiangbo $\mathrm{Ze}^{2, \mathrm{~b}^{*}}$, Yibing $\mathrm{Qu}^{3, \mathrm{c}}$, Han Zhang ${ }^{4, \mathrm{~d}}$ \\ ${ }^{1}$ Jinan City, Shandong Province, China University of Jinan \\ ${ }^{2}$ Jinan City, Shandong Province, China University of Jinan \\ ${ }^{3}$ Jinan City, Shandong Province, China University of Jinan \\ ${ }^{4}$ Jinan City, Shandong Province, China University of Jinan

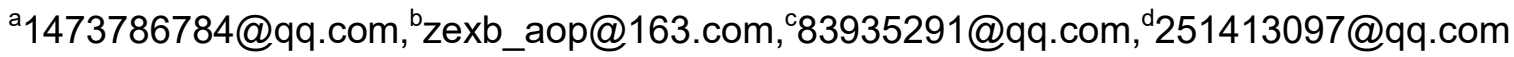

Keywords:Plastic windows; fan regions; two-dimensional convex hulls; computational geometry Abstract: In the computer aided design of doors and windows, the computer only accurate positioning of fan region in the original frame position to further the design of the plastic window. Based on the design software of a door and window, the concept of convex hull in computational geometry is applied to identify the fan regions in the design of plastic steel doors and windows. The convex hull algorithm is used to analyze the frame coordinates to form a convex hull.Using Delaunay triangulation algorithm for convex hull analysis all triangle region The triangulation network is analyzed and merged, and all the fan regions are finally obtained. The convex hull algorithm can overcome the traditional plastic window cannot identify shaped window design software fan area faults, thus greatly expand the scope of design software.

\section{Introduction}

Our enterprises need for rapid design of window type, window type and reduce the repeated design process planning design dimensions necessary and relevant design information. At the same time, the computer can complete the window type automatic design, also must carry on the recognition to all the sector domains in the window type. Therefore, in computer aided design window type, the computer is required to identify the possible fan regions, and the identified sector domains are fed back to the subsequent fan type designation. How to quickly and accurately identify the computer fan frame area is the implementation of computer aided design of windows need to solve the problem.

The window fan region is composed of frame and transom welding to form a closed polygon. The structure of window type graph topology in the computer, using topological graph closed loop algorithm analysis will draw a variety of plastic window closed polygon frame diagram, but these are not the actual fan region graph. As shown in Figure 1, the solid line in the figure represents the circuit that the topology can identify. Therefore, this paper introduces the concept of computing convex hull geometry, the coordinate frame window type abstraction for planar point set; convex hull algorithm was used to analysis the fan area, effectively solves the identification of special windows; in the accurate identification of areas at the same time improve the sash windows design flexibility. 


\section{Fan area introduced}

Plastic fan area introduction. With the development of plastic welding process, welding equipment, plastic frame shape tend to be diversified, in addition to the common square window, there are different shapes of special-shaped windows. The window sash window area is composed of the edge of the frame and the edges are welded into the very. This polygon is defined as the minimum loop, where the minimum loop is used instead of the fan region. The frame is the smallest loop window fan region recognition in the window. As follows, there are four fan regions, i.e., four minimum loops, in Fig. 1.

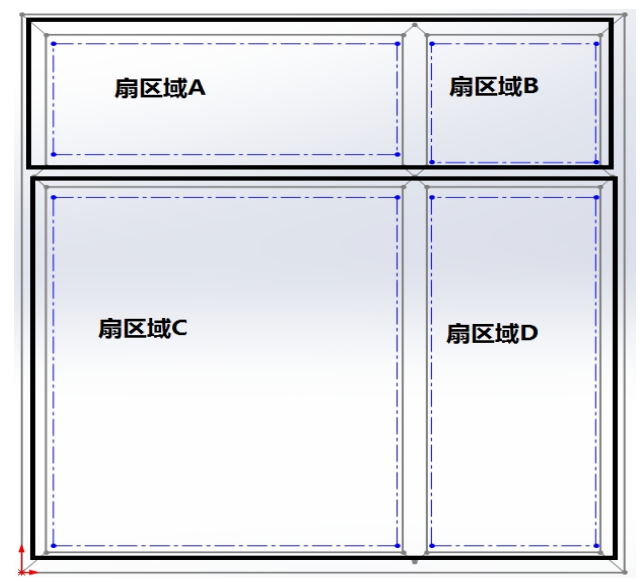

Figure 1 topology can identify the fan area loop

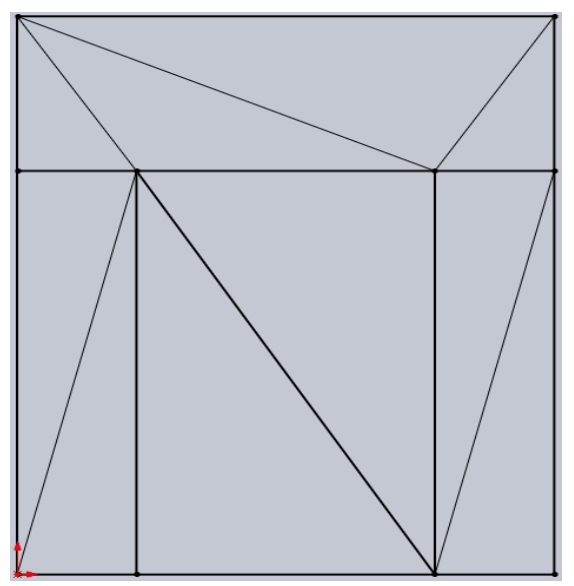

Figure 2 Window triangulation

\section{Process and analysis of the fan region recognition method for plastic window}

Analysis of recognition methods for fan regions .This paper through a API interface window design software acquisition window all frame edge and edge coordinate. The outer frame points form a two-dimensional point set $\mathrm{S}$, so that the point set S.Plastic window frame is a convex polygon, fan area is a closed polygon, from any polygon polygon principle can be divided into n-2 triangles.Thus, Any windows fan area can be obtained by splicing adjacent triangles. For triangulation of point set $S$, the following formula is used to find the third vertex when constructing a triangle:

$$
\cos \mathrm{C}=\frac{\left(\mathrm{a}^{2}+\mathrm{b}^{2}+c^{2}\right)}{2 a b} .
$$

The $\mathrm{a}, \mathrm{b}$ and $\mathrm{c}$ are three linear distances, and the minimum point of $\cos \mathrm{C}$ is the third vertex. According to the formula above, the third vertex is the closest distance between the two known vertices. The triangle is the smallest triangle, and its interior does not contain other vertices in the set of points S. As shown in Figure 2, the window type triangulation effect diagram. At the same time, the triangle is divided into the undirected graph; finally, the common sides of the adjacent triangle are merged, and then the closed polygon is called the window type fan region.

The method of identifying a fan region. Drawn from the above analysis, the minimum window fan area for the outer loop of points. Thus, a series of adjacent triangles are obtained by triangulation of the convex hull G. According to the geometric theory of polygons, these triangles are the smallest units that make up the smallest loop. By combining the triangle according to the rules, all the smallest loops in the convex hull $\mathrm{G}$ can be obtained. Step plastic sashes area identification methods are as follows:

1.Gets the border, side attributes, and endpoint coordinates, forming the $2 \mathrm{D}$ point set $\mathrm{S}$ of the outline point, and the outline edge collection $\mathrm{L}=\left\{\mathrm{L}_{1}, \mathrm{~L}_{2} \ldots \mathrm{L}_{\mathrm{k}}\right\}$. 
2.The convex hull algorithm is used to analyze the plane point set $\mathrm{S}$, and the convex hull $\mathrm{G}$ and the convex hull G outer frame point are obtained.

3.According to the outer convex hull $\mathrm{G}$, set $\mathrm{L}$ to add attributes to stiles or frame edge edge attribute

4.Traverse the triangle collection $\mathrm{T}$, repeat the following operations.

5.Select a triangle $\mathrm{T}_{\mathrm{n}}$, and use the search algorithm to draw all the adjacent triangles and common edges, and create the root node in the undirected graph $\mathrm{G}$.

6.The convex hull $G$ is triangulated to obtain the set $T$ of all triangles, $T=\left\{T_{1}, T_{2} \ldots T_{k}\right\}$.

7.Determine whether the common edge of the adjacent triangle $\mathrm{T}_{\mathrm{b}}$ is the outer edge of the frame, and if not the outer edge of the frame, create the child node in the undirected graph $\mathrm{G}$, and make the $\mathrm{T}_{\mathrm{n}}=\mathrm{T}_{\mathrm{b}}$ jump to step 5 .

8.Traversing undirected graph $\mathrm{G}$, all nodes are merged to obtain the minimum loop $\mathrm{R}_{1}$, stored in the collection of minimum loop set $\mathrm{R}$.

This article uses Python to implement the above algorithm, using the list and dictionary in Python to store the Outline Collection $\mathrm{L}$ and triangle set $\mathrm{T}$. The classical algorithms for constructing convex hull of two-dimensional point set include Graham scan, Jarvis algorithm and divide and conquer algorithm.In the plastic window for a simple polygon and the time complexity of the algorithm, this paper adopts Graham scanning method for constructing convex hull.

\section{Minimum loop identification method of window type convex hull}

Triangulation of window convex hull.This paper adopts irregular triangle net constructed convex polygon $\mathrm{G}$ coverage, the advantages of triangulated irregular network is able to use a random sampling area got the original elevation points directly generate triangular network, build the resolution method triangulation for generating Delaunay triangulation ${ }^{[4]}$. Delaunay triangulation algorithm based on triangulation growth method is adopted in this paper. ${ }^{[5] .}$ At the same time, the LOP optimization algorithm is optimized to generate the triangular mesh. The basic steps of the algorithm are as follows:

1.Determine the first triangle; find the abscissa of the smallest point from the point of the convex hull of $\mathrm{G}$ frame, defined as the first vertex, find the distance with the nearest point point as second vertices, traversing the convex hull $\mathrm{G}$ all points obtained with the first vertex, vertex second and two with the nearest point vertex collinear points as third vertices this, the vertices of the triangle in counterclockwise edge form $\mathrm{P}_{1}, \mathrm{P}_{2}, \mathrm{P}_{3}$ and $\mathrm{P}$ will be added to the collection, in the collection of $\mathrm{T}$ in triangle triangle.

2.Extend a triangle, traverse a line set, $\mathrm{L}$, and repeat the following operations.

3.Select a section $\mathrm{P} 0$ in the set $\mathrm{P}$, traverse all the points of the convex hull, select the right side of the $\mathrm{P}_{0}$ and the nearest point from the two endpoints, as the third vertex of the triangle, form the triangle $\mathrm{T}_{0}$, and add the triangle $\mathrm{T}_{0}$ to the triangle set. While performing LOP optimization.

4.The three points in the counter are generated in the counterclockwise direction. $\mathrm{E}_{1}, \mathrm{e}_{2}, \mathrm{e}_{3}$, where $\mathrm{e}_{3}$ is substantially the same edge as the $\mathrm{P}_{0}$ bit. The traversal of the straight set $\mathrm{P}$ has the same edge as $\mathrm{e}_{1}\left(\right.$ or $\left.\mathrm{e}_{2}\right)$ When you find, add $\mathrm{e}_{1}\left(\right.$ or $\left.\mathrm{e}_{2}\right)$ to the bottom of the line set $\mathrm{L}$.

Triangular mesh combination of window convex hull .Through the above process, we obtain Delaunay triangulation of covering convex hull $\mathrm{G}$ and all triangular set $\mathrm{T}$. In order to get the minimum loop of window type, we need to merge the triangulation. The adjacency relation of all triangles is determined, and the common edges of adjacent triangles are judged. If the common side is not the outer side of the frame, the two triangles are merged. Continue to traverse the adjacent 
triangles and judge until all common edges are outside the frame.In this paper, the adjacency relation is created by the undirected graph. If the two adjacent triangles satisfy the decision condition, the nodes are created for the two triangles, and the two nodes are connected with the creating edge. Traversing all the connected points in an undirected graph, and merging the triangles represented by these points, can form a minimum loop. The specific steps are as follows:

1.Select any triangle $T_{1}$ from the triangle assembly $T$ and set it as the root node in the undirected graph $\mathrm{G}$.

2.Traverse the triangle set $T$, get the adjacent triangle set $T_{n}$, traverse the triangle collection $T_{n}$, and repeat the following operations.

3.Determine whether the common edge is the outer edge of the frame. If the outer edge of the frame is used, the child node is set up in the undirected graph $G$ and the triangle vertex coordinate property is added to the node.

4.Traverse the set $\mathrm{T}$, get the adjacent triangle set, and go through the adjacent triangle set. If the set is not empty, repeat the step 3,4, and if the collection is empty, jump to step 5.

5.Traverse all the nodes in the undirected graph $\mathrm{G}$, merge all the nodes, get the minimum loop $\mathrm{R}_{1}$, and add the set $\mathrm{R}$ to delete the triangles corresponding to all nodes from the set $\mathrm{T}$.

6.Determines whether the set $\mathrm{T}$ is empty; if the set $\mathrm{T}$ is empty, then ends; if the collection $\mathrm{T}$ is not empty, jump to step 1.

\section{Agorithm complexity analysis}

In constructing the convex hull frame, the Graham scanning method needs nlogn times comparison. In generating triangulation, we need to find the best second points and third points in $\mathrm{n}$ points, and the required number of comparisons is $2 n-3$; each edge needs 2 comparisons when expanding edges, and the number of comparisons needs to be $n(n+2 n)$. The triangle when merging a number $\mathrm{S}$ midpoint triangle with the number of points is not much difference, the number of all triangles can be roughly estimated as $\mathrm{N}$, all adjacent edges need to traverse the triangle triangle set $T$, the number of $3 n-6$ times, traversing a triangle adjacent triangles need to traverse the $2 n-3$, most need to traverse the $\mathrm{N}$ second, the comparing times for $\mathrm{n}(3 \mathrm{n}-6+2 \mathrm{n}-3)$.In summary, the total number of comparisons is $8 n^{2}-7 n-3+n \operatorname{logn}$ times, so the worst case complexity is about $\mathrm{O}\left(\mathrm{n}^{2}\right)$.

\section{Conclusion}

This paper proposes regional recognition method CAD window fan design, this method is based on convex hull algorithm, will be transformed into a two-dimensional frame window design points set by the convex hull algorithm of $2 \mathrm{D}$ point set to form a convex polygon. The convex polygon Delaunay triangulation mesh; with special plastic basis triangulation structure will be divided into good, get all the outer fan area. The method can effectively improve the design of flexible plastic window, lay the foundation for the subsequent process planning.

\section{References}

[1]Lingqing.Wang,Zhengyang.Chen,Shuchang.Chen:An improved simple polygon convex algoriyhm.Computer engineering.200-201(2007).p.33

[2]Mao.Peng:Fast convex hull by the realization and its application(2013)

[3]Wenhua.Jin:Simple and fast convex hull algorithm for planar unorganized point set.(Journal of Beijing University of Aeronautics and Astronautics,2009) 
[4]Yonghe.Liu.Data structure and algorithm for automatic generation of Delauny triangulation and adjacency relation.(Journal of henan polytechnic university,2005)

[5]R.C.Lee,S.S.Tseng,R.C.Chang:Introduction to the Design and Analysis of ALgorithms A Strategic Approach(China Machine Press,2007) 